\title{
Influence of the Atmosphere Upon the Precision of Telescope Pointing
}

\author{
By Francis E. Washer and Leo W. Scott
}

\begin{abstract}
The probable error of a single pointing $\left(P E_{s}\right)$ was measured under conditions such that the effect of the air column intervening between observer and target was introduced or eliminated at will. The substantial reduction in $P E_{s}$ for the air-column-eliminated method as compared with the air-column-present method showed that precision in outdoor pointing is definitely limited by the air column. Some approximate computations were made to show that the value of $P E_{s}$ cannot be appreciably reduced by increasing the magnification of the telescope above 20 .
\end{abstract}

\section{Introduction}

In the course of the work done in connection with the Range and Height Finder Project sponsored at this Bureau by the United States Army Ordnance, it became necessary to study the factors affecting the precision of telescope pointing. A range finder is essentially a double-pointing instrument, and consequently it is possible to deduce the error that may be attributed to the purely optical phase of the range finder system from the results obtained with a single telescope. The results of investigations dealing with the precision of pointing for outdoor targets [1 and 2$]^{1}$ and the effect of magnification on the precision of indoor telescope pointing [3] have already been reported. The present study is concerned mainly with showing that the column of air intervening between target and observer places a limit on the ultimate precision that can be achieved and that, furthermore, the gain in precision resulting from increasing the magnification is negligible above a magnification of approximately 20 diameters.

It must be emphasized that the term "pointing," as used in this paper, refers to a type of pointing where all mechanical errors have been eliminated, and the error of pointing found is an attribute of the combination of the optical system, the observer, and the air column intervening between target and observer. It is wholly devoid of such errors as may result from incorrect reading of ver-

${ }_{1}$ Figures in brackets indicate the literature references at the end of this paper. niers and scales, such as exist on transit circles and similar mechanical devices. In addition, the term " $P E_{\mathrm{s}}$ " refers to the probable error of a single pointing about the instantaneous "true" pointing at the time at which it is made, and is a measure of the error of a single pointing determined from a number of independent pointings taken rapidly, and does not contain any appreciable effect of drift.

\section{Method}

When pointings are made on a distant outdoor target, the image-forming light moves through the intervening air and produces an image of the target in the focal plane of the objective of the viewing telescope. In bringing the image of a selected portion of the distant target into coincidence with the intersection of the cross hairs of the telescope, this setting is affected not only by the errors inherent in the combination of optical system and observer's eye but also by any error that may be contributed by the column of air intervening between telescope and target. For example, it is known that the air is at all times in a state of motion, and it is probable that this turbulence may produce small random rapidly changing deviations in the direction of the image-forming light proceeding from the target to the observer. Such deviations, if they exist, would be evidenced by a larger probable error of a single pointing for the condition of air column present than would be obtained for the same target at the same distance if the effect of air column is eliminated. 
In the present experiment, a special target was used for both types of pointing. This target, a sketch of which is shown in figure 1, consisted of a frame 6 feet square with two diagonal pieces intersecting to simulate a cross hair when viewed from a great distance. The frame was provided with an arm pivoted at the top and capable of being swung

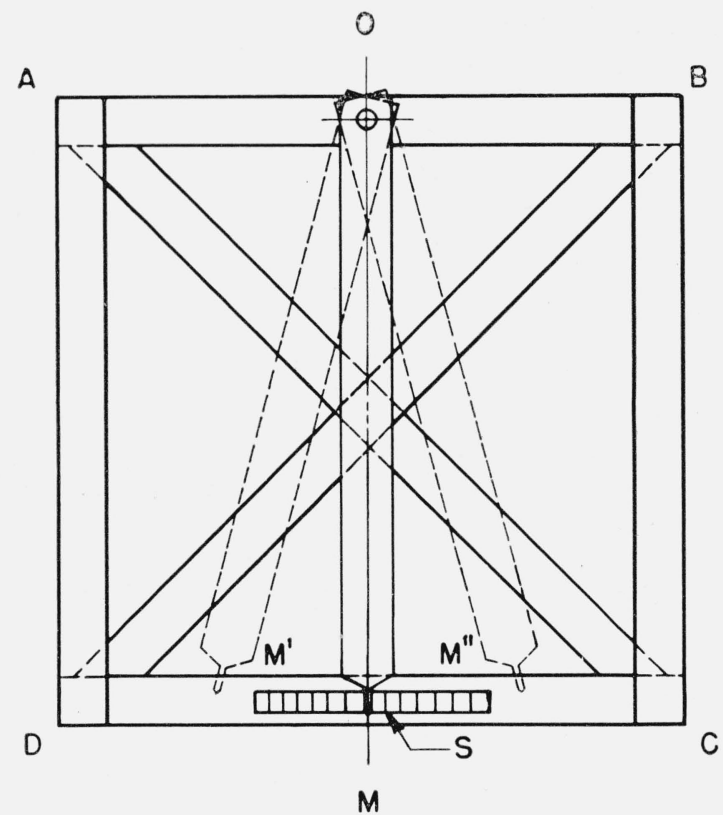

Figure 1. Diagrammatic sketch of special target placed on the tower of Old Soldiers Home.

Intersection of diagonals $A C$ and $B D$ form a cross. The swinging arm, $O M$, pivoted at $O$, is moved to position $M^{\prime}$ or $M^{\prime \prime}$ and then moved into coincidence with the center of the cross under direction of the observer stationed on the grounds of the National Bureau of Standards, distant 4,710 m Successive settings are read with the scale, $S$.

from one side to the other of the intersection of the diagonal pieces. This target was placed in the tower of the Old Soldiers Home and viewed from a station on the grounds of the National Bureau of Standards. The distance separating target and observer is 4,710 meters.

\section{Determination of Pointing Error When Effect of Air Column Is Present}

The vertical member $B C$ of the special target, shown in figure 1, was used as the target in the determination of pointing error with air column present. In this part of the experiment, the apparatus consisted of a telescope equipped with cross hairs and a means by which the observer could vary the pointings at the distant target without disturbing the telescope. This was ac- complished by placing in front of the telescope objective a weak prism capable of rotation about its vertical axis, which is also parallel to the prism axis. As the deviation of a parallel beam of light caused by a weak prism is a function of the angle of incidence on the prism surface, the image of the target can be caused to move from side to side of the telescope cross-hair intersection by oscillating the prism. As the deviation changes slowly with the angle of incidence, this device offers a convenient means for measuring with great precision small variations in the angle of pointing. During the course of an observation the prism was rotated until the image of the target appeared to coincide with the cross hairs, and the angular position of the prism was noted with the aid of an auxiliary telescope and scale in conjunction with a mirror attached to the rotating prism. This reading was converted into seconds in the object space. A series of 10 such observations was taken and the probable error, $P E_{s}$ determined from these data. Several such 10-groups are usually taken in a single run, with a short intermission between each 10-group, and the average $P E_{s}$ is accepted as the value of $P E_{s}$ for the run. The computation of $P E_{s}$ was done in the same manner as reported in a previous paper [3].

All observations and the recording of data, in this part of the experiment, were performed by a single observer, the senior author, which together with the fact that the rotation of the prism was controlled by a smooth rod that must be released between observations, acts to reduce any effect of memory, and so tends to ensure the independence of successive observations.

\section{Determination of Pointing Error When Effect of Air Column Is Eliminated}

In the procedure, described in section II, 1, there can be no question but that an opportunity is afforded the intervening air column to affect the probable error of a single pointing in any manner it may. In this portion of the experiment, the same viewing telsecope was used for observation, the prism was kept immovable, the distance separating target and telesceope was the same, and the cross hairs in the viewing telescope were ignored. The settings were made at the target itself with the aid of a second observer, who moved the swinging arm, $O M$, and brought it into coincidence with the intersection of the diagonal 
bars, $B D$, and $A C$ in accordance with the directions of an observer at the viewing telescope. Under these conditions, the image-forming light proceeding from both the moving and fixed members of the target traversed the same column of air and was subjected to the same conditions before arrival at the viewing telescope, and it is highly improbable that these members changed their apparent relative positions with respect to one another as a result of any effect of the air column intervening between target and viewing telescope. It is true that the image of the target may move about in the focal plane of the objective of the viewing telescope, but because the image moved as a whole, and the coincidence was judged without reference to any fixed mark in the focal plane of the objective, the limit of pointing accuracy was placed by the errors inherent in the optical system, the observer's eye and ability of the second observer to move the arm, $O M$, in accordance with direction, and therefore any effect of the air column was reduced to a minimum. For these reasons, therefore, the effect of the air column was considered eliminated in the determination of the pointing error in this part of the experiment.

To make an observation, an observer in the tower swings the arm on its pivot sufficiently far so that the observer at the telescope can clearly see that the arm and the intersection of the diagonal bars are well separated. The arm is moved slowly to bring it toward coincidence with the intersection point, the movement being stopped when the observer at the telescope considers the moving arm and the intersection of the diagonal bars to coincide. The two observers are in constant telephonic communication throughout the course of a run. The observer in the tower records the setting with the aid of a scale placed at the bottom of the frame. A series of five such settings was made and the probable error, $P E_{s}$, computed thereupon. Several such five-groups were usually taken in a single run, with a short intermission between each five-group, and the average $P E_{s}$ accepted as the value of $P E_{s}$ for the run. Several runs were made for each magnification. The observer at the telescope was the same in both parts of the experiment, so any errors that may arise as a result of eye difference between observers was minimized. At the target distance selected, a movement of $1 \mathrm{~mm}$ at the bottom of the target frame corresponded to an angular displacement of the midpoint of the arm with respect to the intersection of the diagonal bars equal to 0.022 second when viewed from the station where the telescope was located.

\section{Results of Measurements}

In the course of this work, values of $P E_{s}$ for several magnifications were obtained by each method of pointing. Table 1 lists the results of observation, and they are shown graphically in figure 2. It is noteworthy that for all values of magnification curve 1, which shows the value of $P E_{s}$ with effect of air column present, lies above curve 2, which shows the values of $P E_{s}$ with effect of air column eliminated. The failure of the points on curve 1 to lie on a smooth curve arises

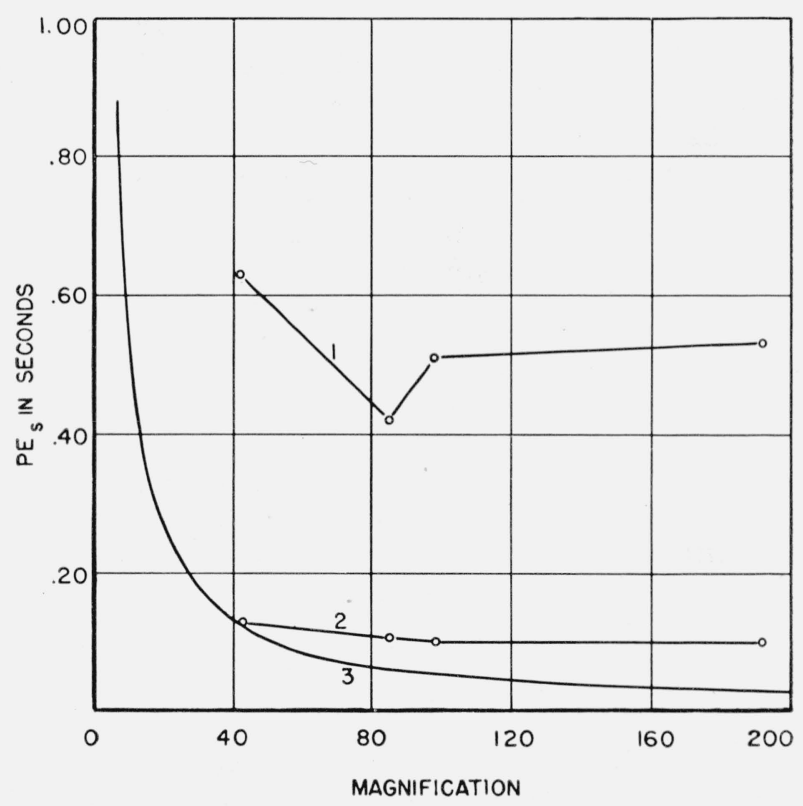

Figure 2. Probable error of a single pointing $\left(P E_{s}\right)$ versus magnification.

The results are for a viewing telescope with an objective of $711-\mathrm{mm}$ focal length, $100-\mathrm{mm}$ aperture. Target is $4,710 \mathrm{~m}$ distant. Curve 1 includes the effect of air column present. For curve 2, the effect of air column is eliminated. Curve 3 is the minimum for the observer on the basis of indoor pointing.

from variations in the atmospheric conditions rather than from any real tendency toward maxima and minima for definite magnifications. In the course of a few days, the spread of values of $P E_{s}$ for a given magnification is as great as these apparent changes from one magnification to another. 
Table 1.-Probable error of a single pointing, $P E_{s}$, as a function of magnification for observations made on an outdoor target with and without the effect of air column

\begin{tabular}{|c|c|c|c|c|c|c|}
\hline \multirow{2}{*}{ Magnification } & \multicolumn{3}{|c|}{ Air column eliminated } & \multicolumn{3}{|c|}{ Air column present } \\
\hline & $P E_{8}$ & $P_{p}$ & $\begin{array}{c}\text { No. of } \\
\text { 5-groups }\end{array}$ & $P E_{s}$ & $P_{p}$ & $\begin{array}{l}\text { No. of } \\
\text { 10-groups }\end{array}$ \\
\hline $42 \ldots$ & $\begin{array}{l}\text { Sec. } \\
0.12\end{array}$ & $\begin{array}{l}\text { Sec. } \\
\pm 0.04\end{array}$ & 8 & $\begin{array}{l}\text { Sec. } \\
0.63\end{array}$ & $\begin{array}{l}\text { Sec. } \\
0.14\end{array}$ & 5 \\
\hline $85 \ldots$ & .10 & .02 & 18 & .42 & .11 & 10 \\
\hline $98 \ldots$ & .09 & .02 & 8 & .51 & .15 & 4 \\
\hline $192 \ldots \ldots \ldots$ & .10 & .01 & 2 & .53 & .07 & 3 \\
\hline
\end{tabular}

A measure of the dispersion of the values of $P E_{s}$ is given in table 1 , under the heading $P_{p}$. Values of $P_{p}$ were obtained by considering each $P E_{s}$ from a single five-group (or 10-group) as an observation and computing the probable error of a single observation from the average value of $P E_{s}$ obtained for a run comprising several fivegroups (or 10-groups). Where several runs are made, $P_{p}$ was the average for all the runs made at a given magnification under the same conditions.

In table 2 the values of $P E_{s}$ are given for observations made on the same day with the same magnifications by each method. It is noteworthy that for magnification 85, for which values are obtained on two different days, the ratio of $P E_{s}$ (air column present) to $P E_{s}$ (air column eliminated) is 2.9 on the first day, whereas on the second day this ratio is 5.3. It is clear that the chief cause of this change in ratio was to be found in the increase of $P E_{s}$ (air column present) from 0.26 to 0.58 , whereas for $P E_{s}$ (air column eliminated) the change was from 0.09 to 0.11 . On the first day, viewing conditions were unusually good, and this was reflected by the low values of $P E_{s}$ by each method. On the second day, visibility was low and turbulence of the air caused considerable unsteadiness in the image of

TABLE 2.-Comparison of $P E_{s}$ for observations made on the same day with same magnification on an outdoor target with and without the effect of air column

\begin{tabular}{|c|c|c|c|c|c|c|c|}
\hline \multirow{2}{*}{ Magnification } & \multicolumn{3}{|c|}{ Air column eliminated } & \multicolumn{3}{|c|}{ Air column present } & \multirow{2}{*}{ Ratio } \\
\hline & $P E_{s}$ & $P_{p}$ & $\begin{array}{c}\text { No. of } \\
\text { 5-groups }\end{array}$ & $P E_{8}$ & $P_{p}$ & $\begin{array}{c}\text { No. of } \\
\text { 10-groups }\end{array}$ & \\
\hline 42 & $\begin{array}{l}S e c \\
0.13\end{array}$ & $\begin{array}{c}S e c \\
\pm 0.06\end{array}$ & 4 & $\begin{array}{c}S e c \\
0.63\end{array}$ & $\begin{array}{c}S e c \\
\pm 0.14\end{array}$ & 5 & 4.8 \\
\hline $85_{\ldots}$. & .09 & .01 & 8 & .26 & .06 & 5 & 2.9 \\
\hline $85 \ldots$ & .11 & .02 & 10 & .58 & .16 & 5 & 5.3 \\
\hline 192 & .10 & .01 & 2 & .53 & .07 & 3 & 5.3 \\
\hline
\end{tabular}

the target. Nevertheless, despite the low visibility, the value of $P E_{s}$ (air column eliminated) increased by only 0.02 second and the value of $P E_{s}$ (air column present) increased by 0.32 second.

In view of the fact that settings made by the air-column-eliminated method did not permit easy control by a single observer, it is worthwhile to consider what values of $P E_{s}$ one might reasonably expect to get by this method with further refinement. Information derived from work done by the senior author on the pointing accuracy of the same telescope on an indoor target [3] was therefore used in the present study. In the course of this work nearly 10,000 observations were made, and from them the following relation between the probable error (in seconds) of a single pointing and the magnification, $M$, of the viewing telescope was derived:

$$
P E_{s}=\frac{4.96}{M}+0.07 \text {. }
$$

It is believed that only the first term of this equation is operative in the present work, therefore values of $P E_{s}$ are predicted from the relation

$$
P E_{s}=\frac{4.96}{\mathrm{M}}
$$

and plotted as curve 3 in figure 2 . It is clear that these values are, for the most part, appreciably lower than those plotted as curve 2. However, in view of the low values of $P E_{s}$ shown in both curves 2 and 3 , it is clear that little gain would result by refining the method.

Furthermore, the values of $P E_{s}$ for the aircolumn-present method cannot be appreciably lowered by extending the number of observations. For here, too, the senior author, assisted by Helen B. Williams, has amassed quantities of data from which it may be deduced that on the average one cannot expect to achieve a value of $P E_{s}$ lower than 0.62 second for pointing at a distant outdoor target with the air-column-present method [1 and 2]. As the air column contributes substantially to the value of $P E_{s}$, it is interesting to consider the possible effect of magnification on outdoor pointing accuracy. In the work on precision of outdoor pointing [1 and 2], the value, $P E_{s}= \pm 0.62$ second, was reported as the value determined for a telescope with magnification of 37 diameters from 4,750 pointings. In the study of the effect of magnification on pointing accuracy for 
indoor targets [3], the value of $P E_{s}$ was found to vary inversely with the magnification. For magnification 37, the contribution of the telescope alone is 0.14 second, neglecting the constant term. The contribution of the air column alone is then

$P E_{s}($ air column $)=\sqrt{(0.62)^{2}-(0.14)^{2}}=0.60$ second .

Clearly then the air column was contributing the major portion of the error in this instance.

If it is assumed that the contribution from the air column was constant on the average, then the approximate effect of magnification on $P E_{s}$ for outdoor targets may be predicted from the equation

$$
P E_{s}=\sqrt{\left(\frac{4.96}{M}\right)^{2}+(0.60)^{2}} .
$$

The values of $P E_{s}$ predicted by eq 4 for a series of magnifications varying from 5 to 100 are listed in table 3. To present a more complete picture, the table lists values for four different values of the air-column contribution. Column 2 gives the values for zero air-column contribution, which is closely approximated in indoor pointing. Column 3 shows the expected values of $P E_{s}$ versus magnification for an air column effect of 0.48 second. This is a close approximation to the air column contribution that may be expected for ranges of 100 to 1,000 meters under average weather conditions. Column 4 shows the expected values of $P E_{s}$ for an air column effect of 0.60 second. This is the average air column contribution that may be expected as an average for all ranges under average weather conditions, or more specifically for the range 1,000 to 4,500 meters. Column 5 shows the expected values of $P E_{s}$ for an air column effect of 0.71 second. This is the average air column contribution that may be expected for ranges in excess of 4,500 meters under average weather conditions.

These results are shown graphically in figure 3 . Consideration of curves 2, 3, and 4 shows clearly that the gain in precision for outdoor pointings for magnifications above 30 diameters is negligible. Even for a magnification as low as 12 diameters, the values of $P E_{s}$ for the three cases illustrated are 31,22 , and 15 percent higher than would be expected for a magnification of 100 diameters. It is of interest to determine the values of the
Table 3.- Predicted values of the probable error, $P E_{s}^{\prime}$, for an outdoor target as a function of magnification

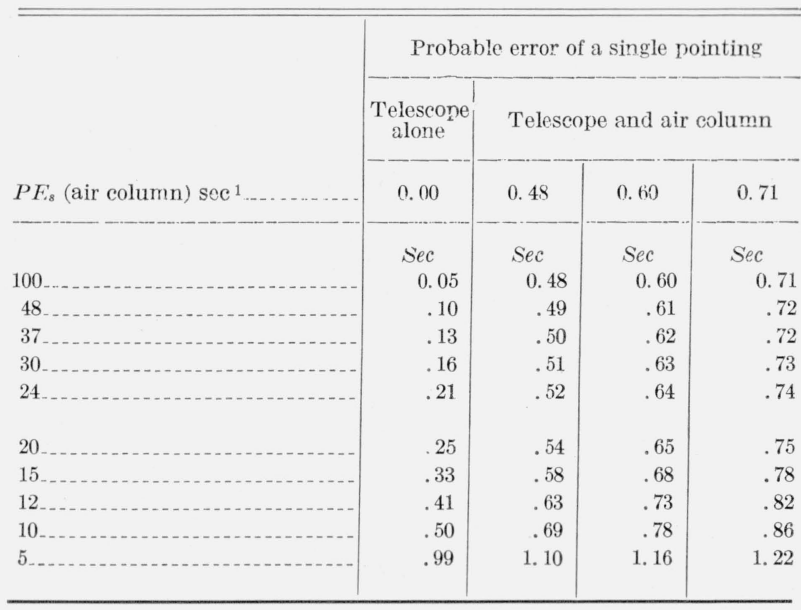

${ }_{1}$ Magnification diameters given in first column.

magnification for which the error for the telescope alone is equal to the error resulting from the air column alone for these three cases. This was done by noting the value of the magnification for which the value of $P E_{s}$ is 1.4 times the value of $P E_{s}$ for the air column alone. For curves 2, 3, and 4 , this condition was satisfied by magnifications of $11,8.5$, and 7.5 diameters, respectively. For magnifications greater than these, the error resulting from the influence of the air column predominates, and relatively small gain in precision of pointing results with increasing magnifi-

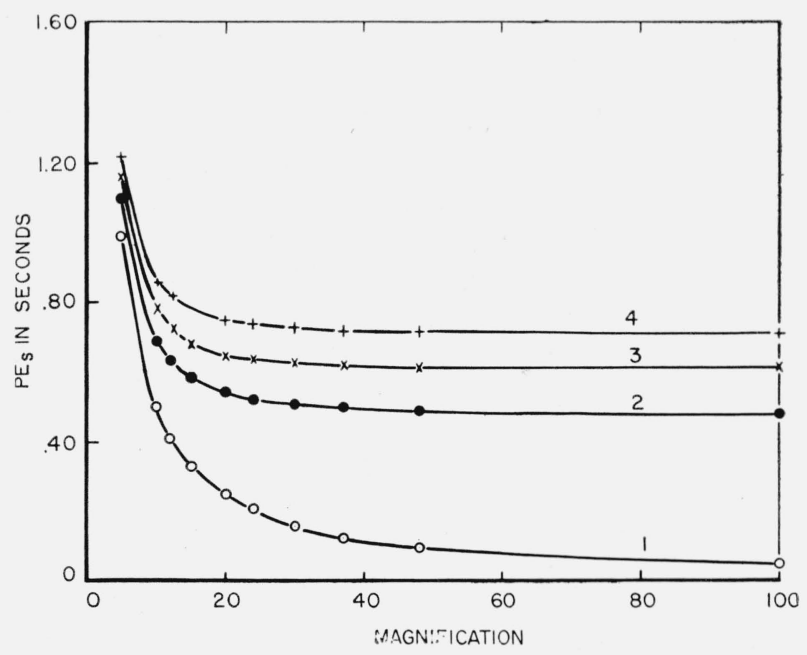

Figure 3.-Probable error of a single pointing $\left(P E_{s}\right)$ versus magnification.

Curve 1 shows the variations of $P E_{s}$ with magnification for air column eliminated. Curves 2,3 , and 4 show the variation of $P E_{s}$ with magnification for errors arising from influence of the air column of $0.48,0.60$, and 0.71 second, respectively. 
cation. For lower values of the magnification the effect of the telescope alone predominates, and appreciable gains in the precision of pointing may be obtained by increasing the magnification until these values are reached.

\section{Conclusions}

The turbulence of the column of air intervening between observer and target is the principal factor that places a limit on the ultimate accuracy in the outdoor pointing of a telescope. The effect of the intervening air column is so great that the lowering of values of $P E_{s}$ that might be expected with increasing magnification is, for all practical purposes, completely masked for all magnifications above 30 diameters. In fact, judging by the values listed in table 3 , it is probable that a magnification of 20 diameters is adequate at least 90 percent of the time in that the average value of $P E_{s}$ for outdoor pointing in the daytime is only 0.04 second higher for magnification 20 than can be expected for a magnification of 100 diameters.

\section{References}

[1] E. E. Washer and H. B. Williams, J. Opt. Scc. Am. 36, 400 (1946).

[2] F. E. Washer and H. B. Williams, J. Research NBS 36, 479 (1946) RP1717.

[3] F. E. Washer, J. Research NBS 39, 163 (1947) RP 1820.

Washington, June 24, 1947. 